\title{
New Methodology for Achieving Trim Condition for a small Flapping Wing Air Vehicle
}

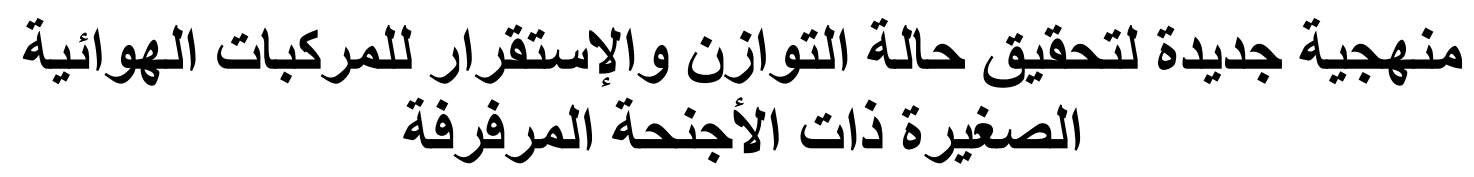

Mohammed A. Fanni ${ }^{\text {a }}$, M. Samy El-Gayyar ${ }^{\text {, }}$, Ahmed Sameh

a Assoc.Prof., Dept. of Mechanics and Robotics, Egypt-Japan University and Technology (E-Just), Alexandria, on leave, prod. Eng. \& Mech. Design, Dept., Mansoura University.

b Assoc. Prof., Prod. Eng. \& Mechanical Design Dept., Mansoura University

c A demonstrator, B. Sc. of Prod. Eng. \& Mechanical Design Dept., Mansoura University

\begin{abstract}
ملخص أنص

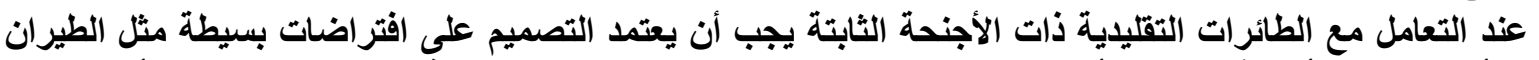

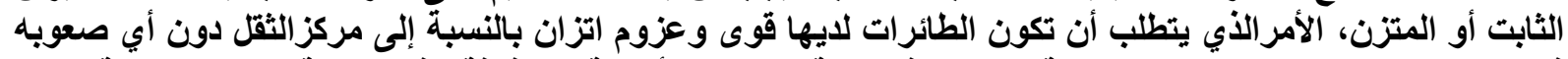

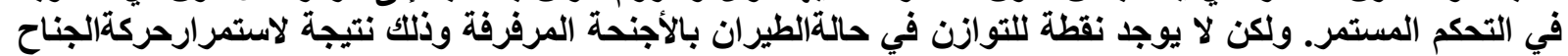

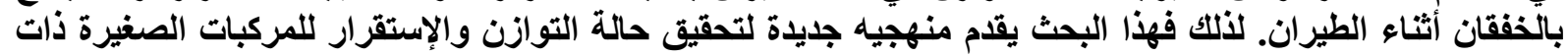

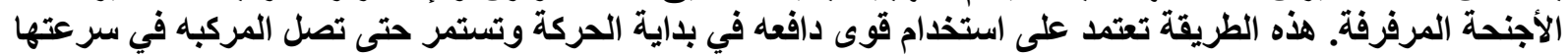

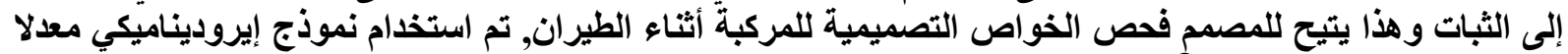

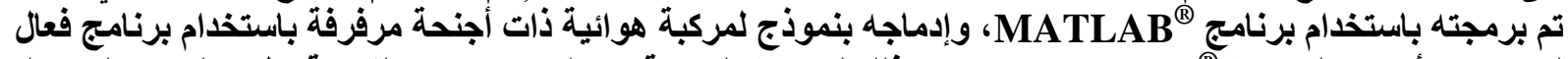

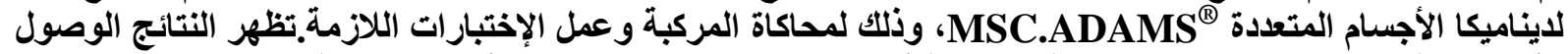

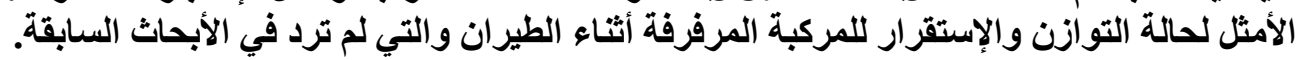

\section{Abstract}

When dealing with a conventional aircraft, the design must be based on simplification assumptions, such as a cruising flight or a trimmed flight condition, which requires aircraft to have forces and moments equilibrium with respect to the center of gravity without any continuous control efforts. However, in case of ornithopters, there is no such equilibrium point due to the continuous flapping wing motion during the cruising flight. Therefore, this paper introduces a new methodology to obtain trimmed flight condition for a small flapping wing air vehicle. This methodology is based on applying initial impulsive forces to the air vehicle in such a way that the flapping wing air vehicle reaches almost constant flight speed after removing the initial impulsive forces. This enables the designer to examine the design parameters of the vehicle during realistic flight performance. A refined aerodynamic model programmed with MATLAB ${ }^{\circledR}$ is integrated into a flapping vehicle model using efficient multi body dynamics software MSC.ADAMS ${ }^{\circledR}$ to test the system. The results show perfect trimmed flight condition for a flapping wing air vehicle that never presented before in the literature.

\section{Keywords}

Flapping flight; Multi-body dynamics; Flight simulation; MSC.ADAMS®; Trim condition.

\section{Nomenclature}

$\omega$ : The angular velocity.

$c$ : The wing's reference chord.

$\beta$ : the section's instantaneous dihedral angle. $\theta$ : The pitch angle of the chord with respect to the free stream velocity.

$\mathrm{h}$ : The plunging displacement.

$\bar{\theta}$ : The section's mean pitch angle.

$\alpha_{0}$ : The section's zero-lift line. 
$\overline{\theta_{\mathrm{a}}}$ : The pitch angle of the flapping axis with respect to $U$.

$\mathrm{U}$ : The relative wind velocity.

$\alpha$ : The relative angle of attack at the $3 / 4$ chord location due to the wing's motion.

'́ : The flow's relative angle of attack at the $3 / 4$-chord location.

$\alpha_{\text {stall }}$ : The stalling angle of attack.

$\mathrm{d}_{\mathrm{Nc}}$ : The normal force due to circulation.

$\rho$ : The atmospheric density.

dy : The width of the wing section.

$\mathrm{Cn}(\mathrm{y})$ : The normal force coefficient.

$\mathrm{d}_{\mathrm{Na}}$ : The normal apparent mass force.

$\dot{\mathrm{v}}$ : The rate of change of the midchord normal velocity component due to the wing's motion.

$d_{N}$ : The section's total attached flow normal force.

$\left(d_{\mathrm{Nc}}\right)_{\text {sep }}$ : The separated normal force due to circulation.

$(\mathrm{Cd})_{\mathrm{cf}}$ : The crossflow drag coefficient.

$\hat{\mathrm{v}}$ : Resultant of the midchord velocity.

$\mathrm{V}_{\mathrm{x}}$ : The midchord chordwise velocity component.

$\mathrm{V}_{\mathrm{n}}$ : The midchord Normal velocity component.

$\left(d_{\mathrm{Na}}\right)_{\text {sep }}$ : The separated normal apparent mass force.

$\left(d_{N}\right)_{\text {sep }}$ : The section's total separated flow normal force.

$d_{D_{\text {camber }}}$ : The chord wise force due to camber.

$\mathrm{d}_{\mathrm{T}_{\mathrm{s}}}$ : The leading edge suction force.

$\zeta_{s}$ : The leading edge suction efficiency factor.

$\mathrm{d}_{\mathrm{Df}}$ : The chordwise friction drag force.

$(\mathrm{Cd})_{\mathrm{f}}$ : The drag coefficient due to skin friction.

$\mathrm{d}_{\mathrm{fx}}$ : The totally separated flow force.

$\mathrm{dL}$ : The instantaneous lift force.

$\mathrm{dT}$ : The instantaneous thrust force.

$\mathrm{f}$ : The flapping frequency.

\section{Subscripts}

Nc : Normal circulatory.

Na : Normal apparent.

Ts: leading edge suction.

Sep: Separated condition.

Df: Friction drag.
$\mathbf{F x}$ : chordwise force.

\section{Superscripts}

- : Mean value

$\cdot$ : Time derivative.

\section{Introduction}

The flapping flight of birds, bats and insects has fascinated many researchers in various fields such as biology, zoology, aerodynamics and electronics because of their highly efficient maneuverability and aerodynamic benefits especially in a low Reynolds number flight regime [1].For many centuries, numerous efforts have been made to mimic nature's fliers in order to make artificial flapping wing vehicle. It seemed that the flapping vehicles are being forgotten with the great success of fixed wing aviation. However, the rebirth of flapping flying machines is seen in much smaller devices, namely flapping unmanned vehicles (UAV), equipped with a small video camera and various sensors. They can be used for surveillances and rescue missions [2]. Many analytical and experimental studies on flapping wings have also been performed to understand the aerodynamic characteristics and flight mechanisms of the flapping wings [3]. Most previous works seem to fall roughly into two categories. The first, and most common, is the quasi-steady model where unsteady wake effects are ignored. That is, flapping frequencies are assumed to be low enough that shed effects are negligible. Such assumption gives a great simplification to the aerodynamic modeling [3]. The second category accounts for unsteady aerodynamic effects by modeling the wake in variety of ways, induced by unsteady wing motion and vortices shed from leading edges.

In the present study, an aerodynamic model is suggested based on modified strip theory and improved to consider high relative angle of attack and dynamic stall effects due to pitching and plunging motions [4]. The aerodynamic forces applied on the vehicle wings are 
programmed using MATLAB $^{\circledR} /$ Simulink. A multi-body dynamics software MSC.ADAMS $^{\circledR}$ is used to model the full vehicle parts (wings, body, mechanism and tail). The forces from MATLAB ${ }^{\circledR}$ are then transferred into MSC.ADAMS ${ }^{\circledR}$ through MATLAB/ADAMS interface. Since the aerodynamic model assumes constant flight speed, it is necessary to reach a near constant flight speed in the flight simulation. After reaching such speed, both aerodynamic model and dynamic simulation become justified and hence the examinations of the design parameters become possible. Iterations are performed to obtain the trim condition to have a stable flapping flight with small fluctuations in forward velocity, pitch angle and altitude at a specified frequency using initial impulse forces.

\section{Aerodynamic modeling of Flapping wing}

The computational aerodynamic model used in this study is based on the modified strip theory, in which the wing is divided into thin strips. Each strip is considered an aerofoil of finite width as shown below in figure1. The instantaneous aerodynamic forces are computed for each section and then integrated over the entire wingspan. The advantages of this model are the ability to account for vortex-wake effects, camber forces, leading edge suction effect and post stall behavior. The kinematic for each section of the wing is illustrated below in figure 2. The section's motion consists of a plunging velocity, $\dot{h}$ and a pitch angle, this model suggested a routflapping kinematics with no spanwise bending.

Therefore, the section's circulatory normal force is given by

$$
d_{N c}=\frac{\rho u v}{2} \operatorname{Cn}(y) \cdot C \cdot d y
$$

$v$ : The flow's relative velocity at $1 / 4$ chord location, and

$$
\operatorname{Cn}(y)=2 \pi\left(\bar{\alpha}+\alpha_{0}+\bar{\theta}\right)
$$

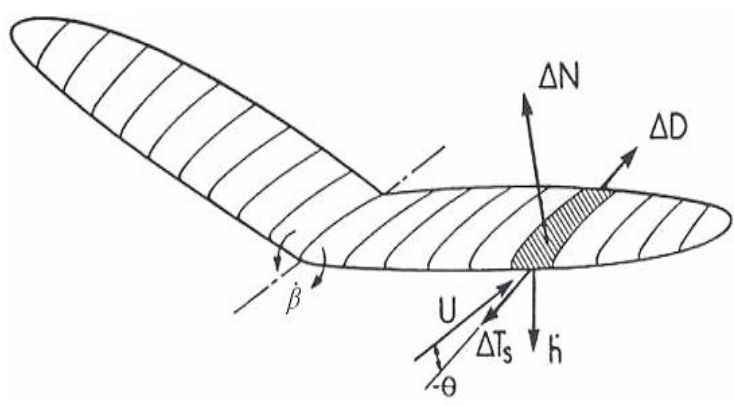

Fig.1. Root flapping wing and aerodynamic

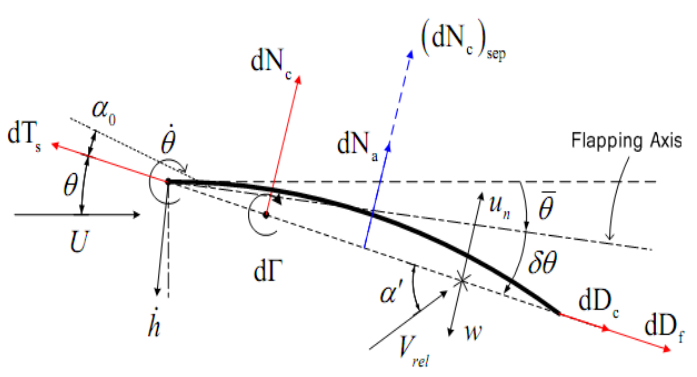

Fig.2. Wing section aerodynamic forces.

Where $\rho, u, \alpha_{0}, \bar{\alpha}$ are the atmospheric density, the flight speed, and the angle of section's zero lift line and the remaining angle $\bar{\alpha}$ can be computed in details in DeLaurier [3] based on the relative angle of attack at the $3 / 4$ chord $\alpha$ due to the wing's motion and the downwash velocity [5, 6 and 7].

Normal force contribution comes from the apparent mass effect which acts at the mid chord and it is given by

$$
d_{N a}=\frac{\rho \pi c^{2}}{4} \dot{v}_{2} d y
$$

Where $\dot{v}_{2}$ is the time rate of change of the mid chord normal velocity component due to the wing's motion.

Therefore, the section's total attached flow normal force is

$$
d_{N}=d_{N a}+d_{N c}
$$

The section's circulation distribution likewise generates forces in the chordwise direction.

From DeLaurier [3], the chordwise force due to camber is given by 


$$
d_{D_{\text {camber }}}=-2 \pi \cdot \alpha_{0}(\bar{\alpha}+\bar{\theta}) \cdot \frac{\rho u v}{2} c d y
$$

The leading edge suction force [8] is obtained by

$$
d_{T_{S}}=\zeta_{s} \cdot 2 \pi\left[\bar{\alpha}+\bar{\theta}-\frac{1}{4} \frac{c \dot{\theta}}{u}\right] \cdot \frac{\rho u v}{2} \cdot c \cdot d y
$$

The chordwise frictions drag force due to viscosity [9].

$$
d_{D f}=(C d)_{f} \cdot \frac{\rho v^{2} x}{2} \cdot c \cdot d y
$$

Therefore, the total chordwise force is given by

$$
d_{f x}=d_{T_{s}}-d_{D_{\text {camber }}}-d_{D f}
$$

The criterion for attached flow over the section [10] is assumed as follows

$$
\begin{aligned}
&\left(\alpha_{\text {stall }}\right)_{\min } \leq {\left[\bar{\alpha}+\bar{\theta}-\frac{1}{4} \frac{c \dot{\theta}}{u}\right] } \\
& \leq\left(\alpha_{\text {stall }}\right)_{\max }
\end{aligned}
$$

Where the term in the bracket represents the dynamic stall effect induced by pitching motion, when the attached flow range is exceeded, the flow is assumed to be totally separated and all the chordwise force are negligible as localized post stall behavior.

The normal force in the stall condition are given by

$$
\begin{aligned}
& \left(d_{N c}\right)_{s e p}=(C d)_{c} \cdot \frac{\rho \hat{v} v_{n}}{2} \cdot c \cdot d y \\
& \left(d_{N a}\right)_{s e p}=\frac{\rho \pi c^{2}}{8} \dot{v}_{2} d y
\end{aligned}
$$

Where $\hat{v}$ and $v_{n}$ are the resultant flow velocity and the normal flow velocity at midchord location respectively.

Finally, the section's instantaneous lift and thrust can be computed as follows

$$
\begin{aligned}
& d L(t)=d N \cos \theta+d F_{x} \sin \theta \\
& d T(t)=d F_{x} \cos \theta-d N \sin \theta
\end{aligned}
$$

The aerodynamic forces of the sections can be integrated along the span to obtain the whole wing's instantaneous lift and thrust as follows

$$
L(t)=2 \int_{0}^{b / 2} \cos \beta d L
$$

$T(t)=2 \int_{0}^{b / 2} d T$

Where $\beta$ is the section's instantaneous dihedral angle.

\section{Modified aerodynamic model}

According to the flight condition, if the plunging velocity is increased or the free stream velocity relative to $\dot{h}$ is decreased, the wing section can be exposed to a high relative angle of attack, so the assumption of small relative angle of attack is not valid [2]. To consider high relative angle of attack, the aerodynamic model is improved, using the horizontal and vertical components of the velocity at $1 / 4$ chord location, the relative angle of attack can be defined as follows:

$\gamma=\tan ^{-1}\left[\frac{\dot{h} \cdot \cos \left(\theta-\overline{\theta_{0}}\right)-\omega+0.25 \cdot C \cdot \dot{\theta}+u \sin \theta}{u \cos \theta-\dot{h} \cdot \sin \left(\theta-\overline{\theta_{a}}\right)}\right]$

The section's aerodynamic forces can be expressed as follows

$$
d_{N c}=2 \pi\left(\bar{\alpha}+\alpha_{0}+\bar{\theta}\right) \cdot \cos \gamma \cdot \frac{\rho u v}{2} C \cdot d y
$$

$d_{T_{S}}=$

$$
\zeta_{s} \cdot 2 \pi\left[\bar{\alpha}+\bar{\theta}-\frac{1}{4} \frac{c \dot{\theta}}{u}\right] \cdot \sin \gamma \cdot \frac{\rho u v}{2} \cdot c \cdot d y
$$

$d_{D_{\text {camber }}}=$

$-2 \pi \cdot \alpha_{0}(\bar{\alpha}+\bar{\theta}) \cdot \cos \gamma \cdot \frac{\rho u v}{2} c d y$

Other aerodynamic forces, $d_{N a}$ and $d_{D f}$ are the same in the modified strip theory developed by DeLaurier [3]. The effects due to the dynamic stall can be generated by the plunging motion as well as the pitching motion. Using dynamic stall effect and high relative angle of attack, in this study three kinds of flow conditions are used as follows:
(1) - attached flow condition
$\left(\alpha_{\text {stall }}\right)_{\min } \leq \gamma \leq\left(\alpha_{\text {stall }}\right)_{\max }$
(2) - dynamic stall condition

$\left(\alpha_{d y}\right)_{\min } \leq\left[\gamma-\frac{3}{4} \frac{c \dot{\theta}}{u}\right] \leq\left(\alpha_{d y}\right)_{\max }$ 
(3)- Post stall condition: over the dynamic stall range.

In the attached flow range and post stall flow range, the aerodynamic forces are the same as those defined in the modified strip theory. In the dynamic stall range, the aerodynamic forces are applied as those in attached flow, but only the direction of the leading edge suction force is assumed to be perpendicular to the effect due to the leading edge vortex [4].

\section{Modeling and Simulation}

In order to have a successful and an efficient flight simulation of the flapping vehicle and obtain accurate results, the commercial software MSC.ADAMS ${ }^{\circledR}$ is used to model the wing kinematic motion.

\subsection{Mechanism selection}

The linkage mechanism has to be powered by an electric motor; the rotational motion of the shaft has to be converted to a harmonic flapping motion with the constraint of simplicity of the mechanism. The used mechanism is basically a four bar crank rocker mechanism, the wing is attached to the rocker, this mechanism doesn't give asymmetric flapping motion neither it would give a motion that is fully harmonic. The main advantages of this mechanism are the simplicity and it would be very light. Figure 3 shows the simple four bar mechanism used in the model.
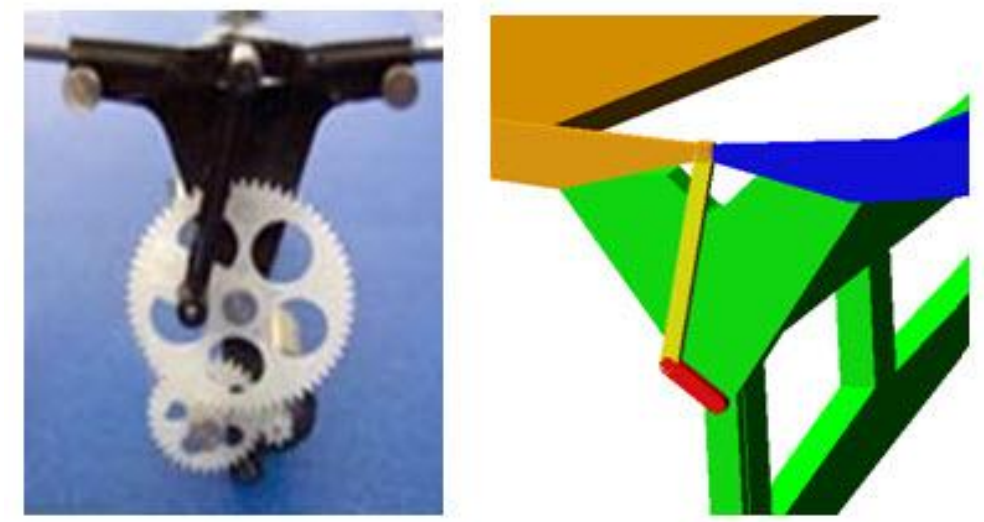

Fig.3. A simple Four bar flapping mechanism

\subsection{Wing kinematics}

Each wing is divided into 12 sections as shown in figure 4 below, each division has all the aerodynamic forces which will be integrated along the wingspan to have the full instantaneous aerodynamic lift and thrust forces. The total weight of the model is about 60 grams as the model consists of the flapping mechanism, motor, two wings, tail and the main body which will contain

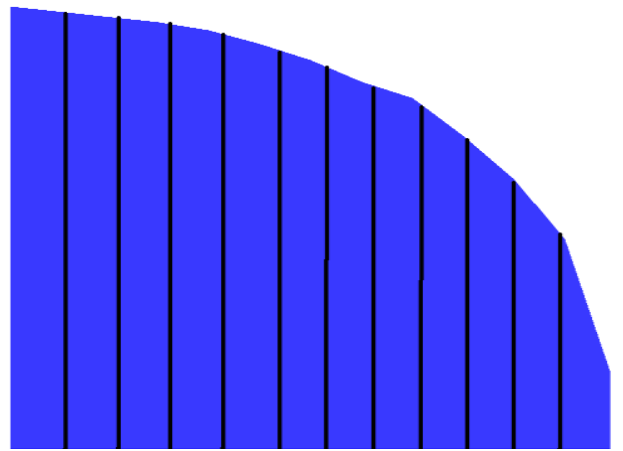

Fig.4. Elliptical wing shape used in the simulation. the control board and the battery. The wingspan is about $30 \mathrm{~cm}$. After constructing the model, we accurately determine the locations and dimensions of the aerodynamic forces for each section of the wing; each section would have eight aerodynamic forces as shown in figure 5 below, with approximately 192 force vectors for the entire model, both wings.

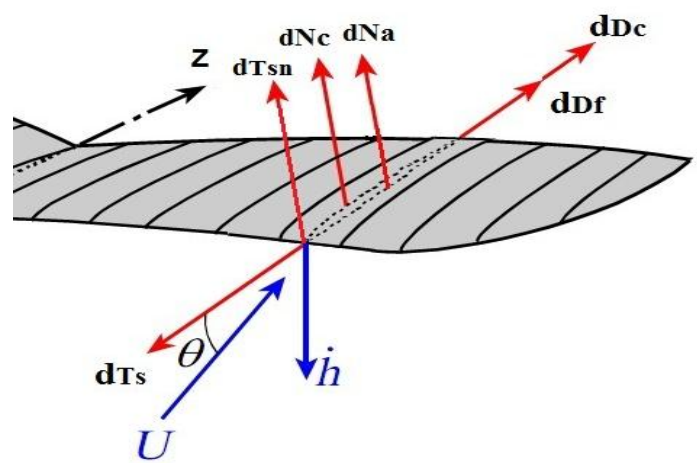

Fig.5. Applied Forces on the aerofoil. 
Using the plug-in built in MSC.ADAMS ${ }^{\circledR}$ which named Adams/control; interaction between MSC.ADAMS ${ }^{\circledR}$ and MATLAB ${ }^{\circledR}$ is performed to make a program containing all the aerodynamic forces equations. The output forces from MATLAB ${ }^{\circledR}$ will be the inputs imported in MSC.ADAMS ${ }^{\circledR}$, so the simulation can be performed accurately and fast. Through MATLAB ${ }^{\circledR} /$ Simulink, a simple user interface is built in which all the simulation and aerodynamic parameters required are submitted. Also, the different model wing's dimensions and sections' chord length can simply changed through Simulink interface. A flow chart showing the main steps of modeling and simulation is shown in figure 6 .

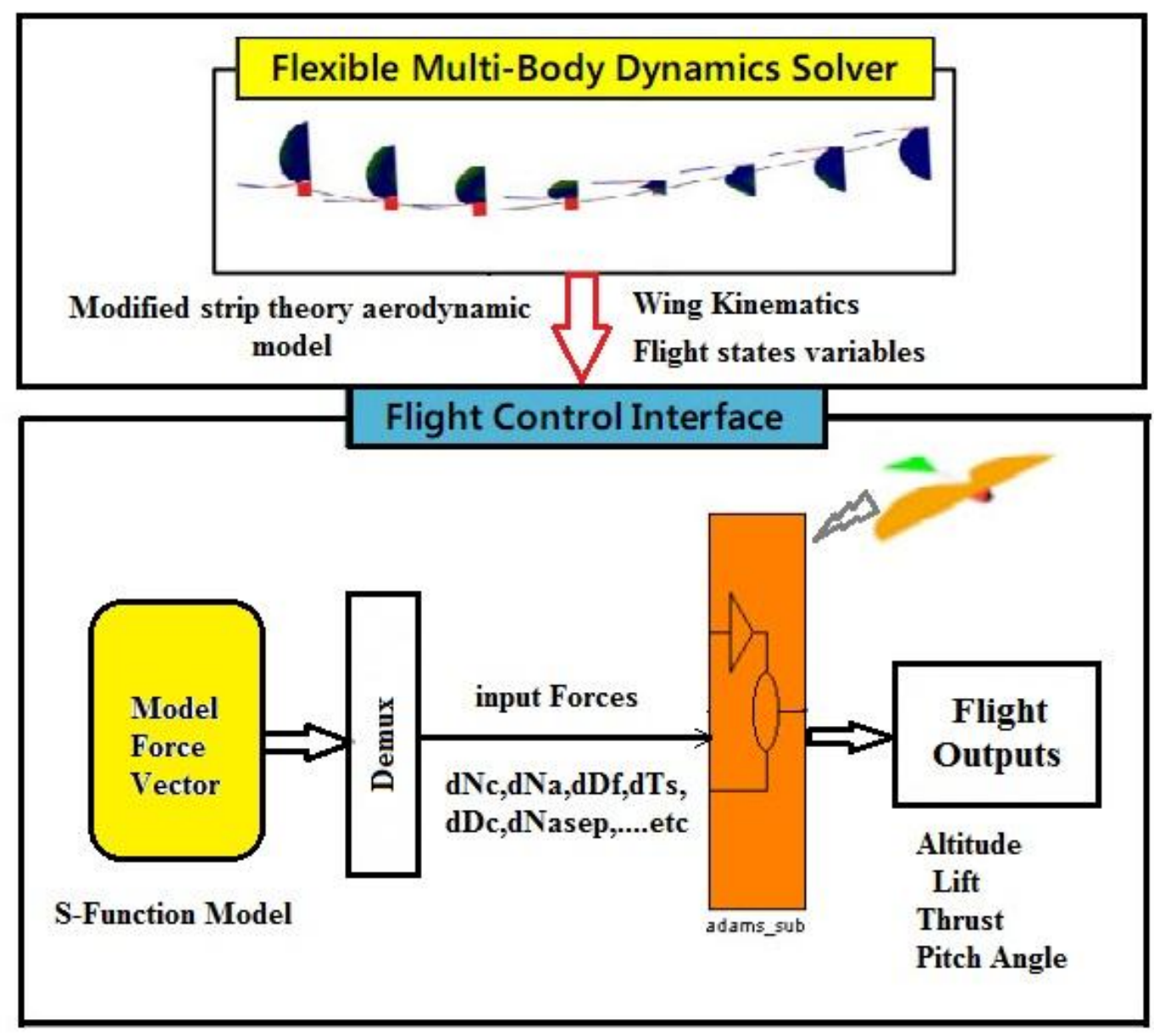

Fig.6. A schematic diagram shows the sequence of simulation.

\section{Results and Discussion}

In this study, a time-efficient integrative simulation frame work for the trimmed longitudinal flight of model ornithopter is performed accurately. The trim condition main parameters in this model are frequency, pitch angle and the relative forward velocity measured from the center of gravity of the model. Obtaining the trimmed flight parameters is based on trial and error method, an initial guess of frequency of wings, forward velocity and the pitch angle is considered. Starting the iterations based on trial and error with ditterent trequencies startıng from $5 \mathrm{~Hz}$ to $20 \mathrm{~Hz}$ for our small model with maximum weight of 60 grams, and also we tried for different pitch angles starting from 0 to 20 degrees. The main parameter is the forward velocity or in another word the relative wind velocity as the trim condition occurs at a velocity that has small fluctuations and seems to be constant or near constant during flight, so velocities are considered to start from $0.1 \mathrm{~m} / \mathrm{s}$ to $20 \mathrm{~m} / \mathrm{s}$.

At a pitch angle of $7.5 \mathrm{deg}$, velocity of 11 $\mathrm{m} / \mathrm{s}$ and frequency equal to $13 \mathrm{~Hz}$, the model raised and has a sufficient lift 
almost greater than the weight and gives a slow raising flight altitude with a rate of 7 $\mathrm{cm}$ per sec but we failed to achieve a constant forward velocity. Inserting an impulsive force within a small working time about $0.07 \mathrm{sec}$ is then suggested in order to help the model to achieve the trim condition and perform a stable flight. Using several values of impulsive forces all applied in the direction of the forward velocity of the model; we noticed that at a start force of 100 Newton, we succeeded to obtain the best stability and performance of trimmed flight condition as the ornithopter has a stable altitude at 7.84 meters above ground after 1 second and achieves the best equilibrium condition in which lift almost equals to the weight of ornithopter. For forward velocity, we get fluctuations in the first second of launching and rapidly decrease to very small fluctuations after 1 second to have a constant velocity of 11.4 $\mathrm{m} / \mathrm{s}$. The pitch angle also has small fluctuations varying from 7.4753 to 7.5000 degree as shown in figure 7.

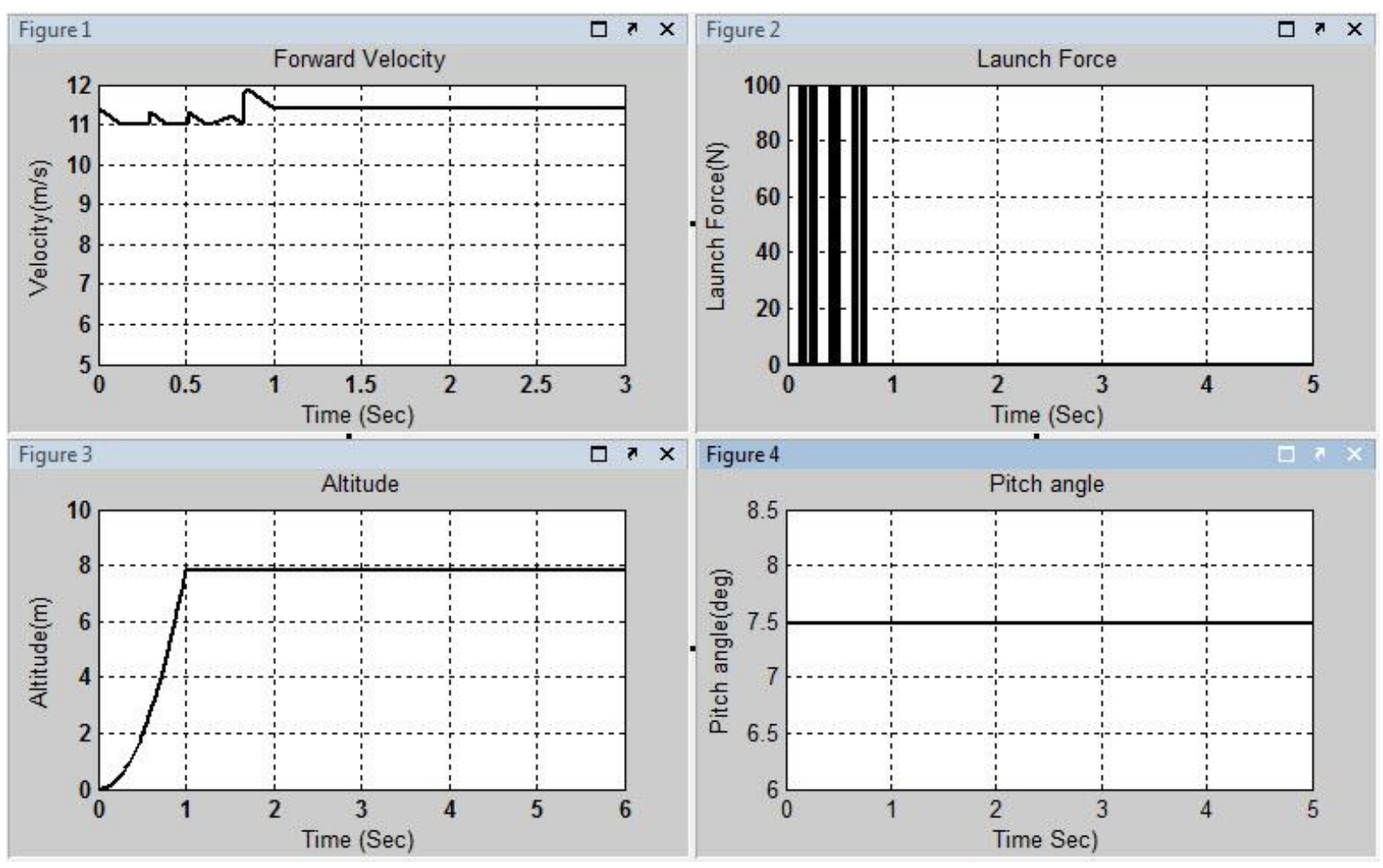

Fig.7. Trim condition obtained at impulsive force $100 \mathrm{~N}$, pitch angle $7.5 \mathrm{deg}$, velocity $11 \mathrm{~m} / \mathrm{s}$ and altitude about $7.84 \mathrm{~m}$.

By increasing the impulsive force, we succeeded to obtain the trim condition at a force of $10000 \mathrm{~N}$ as the model has small fluctuations at altitude about 360 meters after 6.8 seconds and have a constant velocity of $11.1 \mathrm{~m} / \mathrm{s}$. At a start force of $20000 \mathrm{~N}$ we obtain the trim condition after 10.6 seconds at altitude of about 900 meters and a constant velocity of $11.2 \mathrm{~m} / \mathrm{s}$ as shown below in figure 8 . 

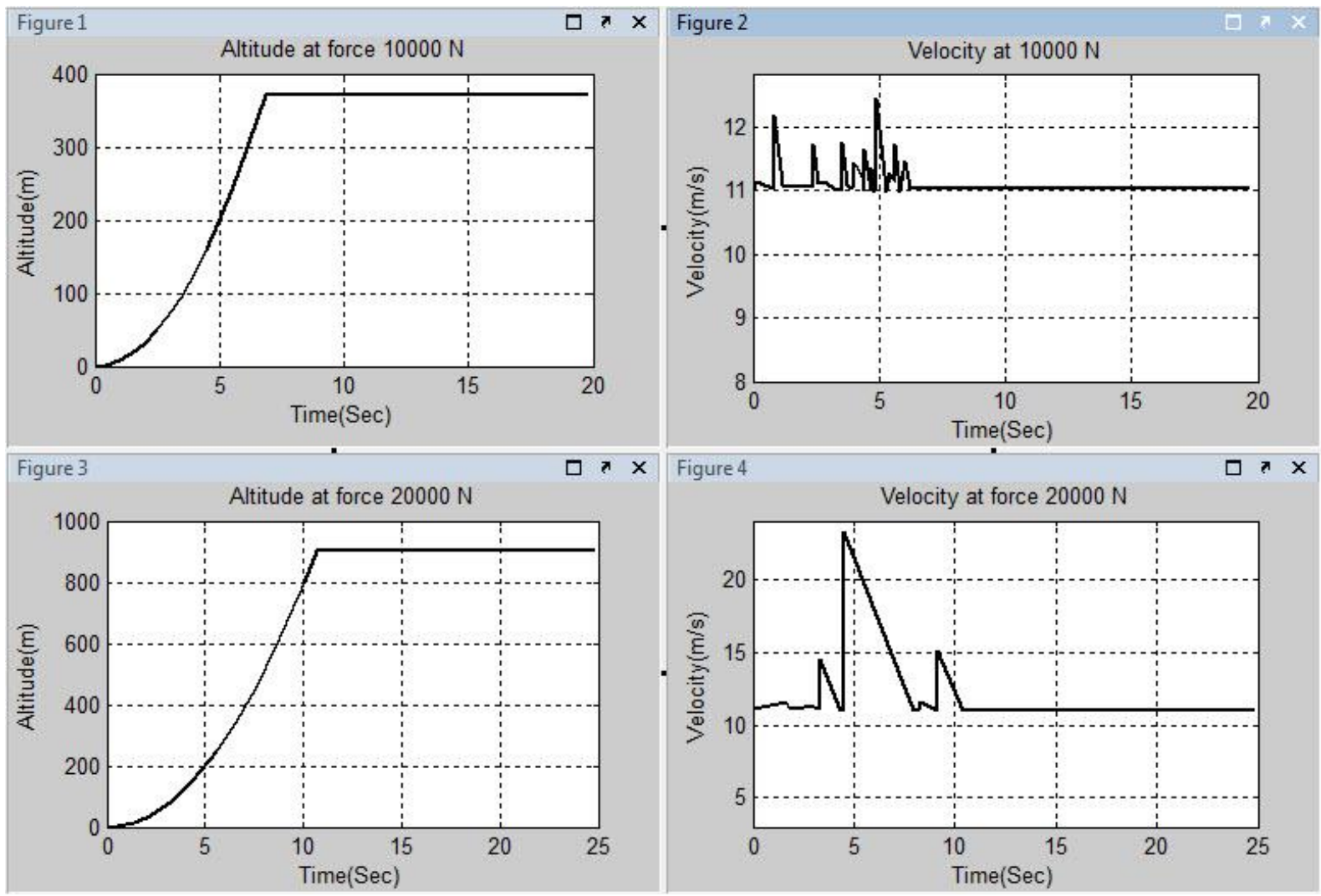

Fig.8. Trim condition obtained at impulsive forces 10000 and $20000 \mathrm{~N}$.

By increasing the start force, we obtain the trim condition at 50000 and $60000 \mathrm{~N}$ after a time about 5 and 22 seconds respectively, the altitude is about 180 and 3500 meters respectively, the forward velocity has a constant value at $11 \mathrm{~m} / \mathrm{s}$ at both 50000 and $60000 \mathrm{~N}$ as shown below in figures 9 and 10.

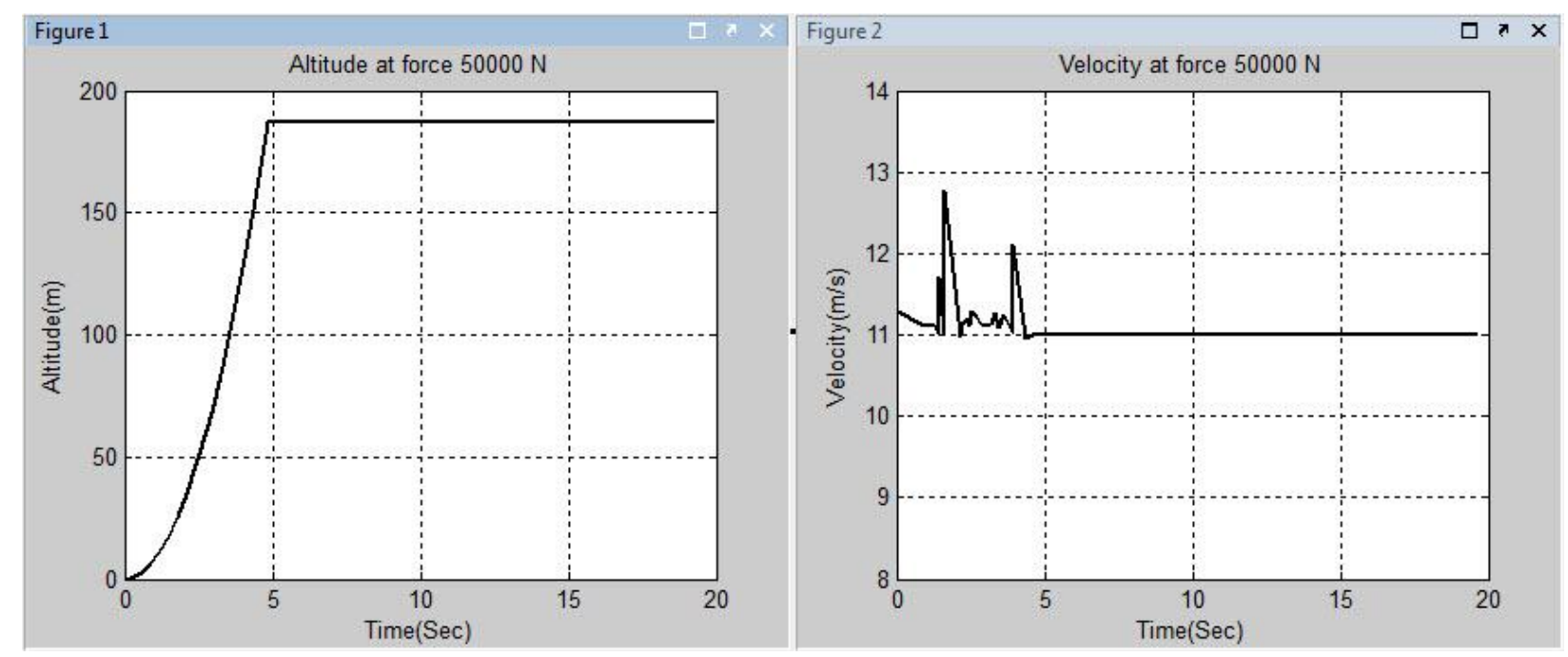

Fig.9. Trim condition obtained at impulsive force $50000 \mathrm{~N}$. 

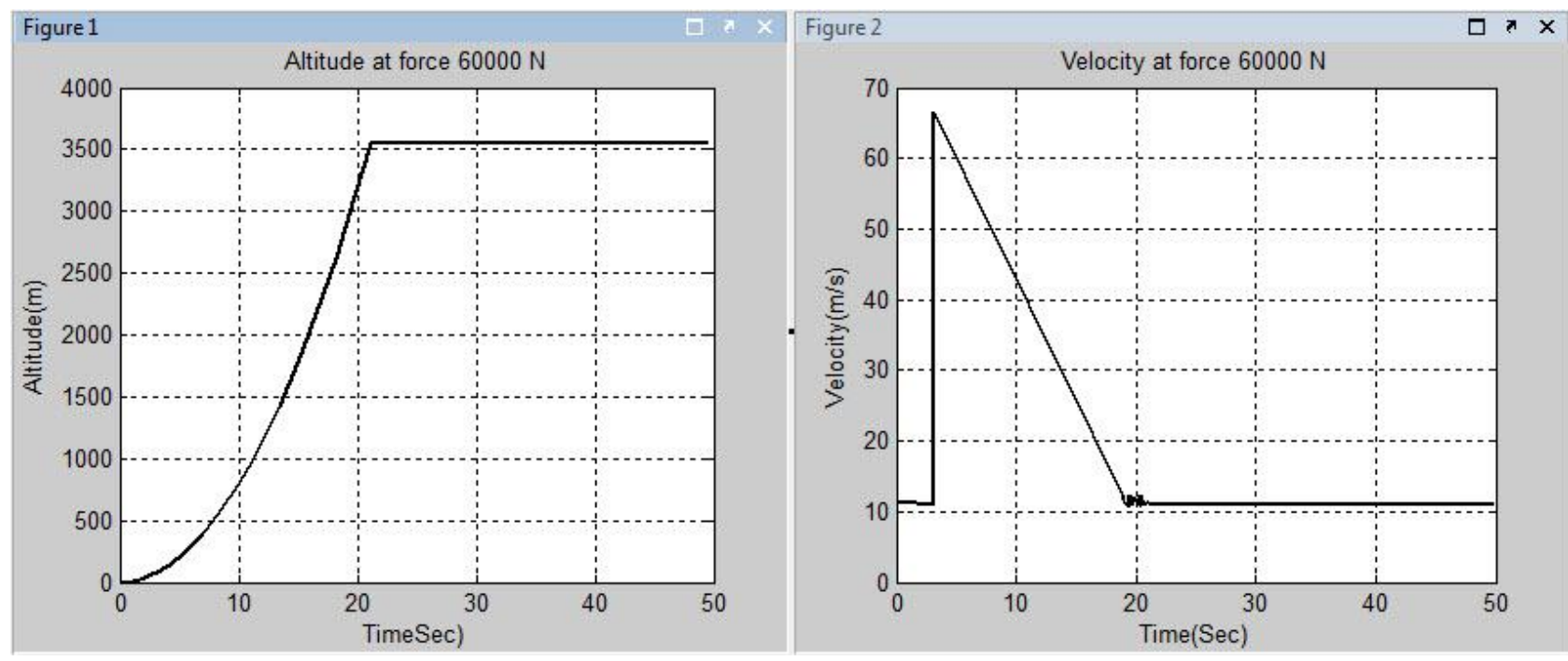

Fig.10. Trim condition obtained at impulsive force $60000 \mathrm{~N}$.

Many iterations are performed for different values of flight parameters without using impulsive forces but the trim condition only achieved using impulsive forces with specified values. Figures 11 and 12 show some of these unsuccessful tries to obtain the trim condition but the results as shown didn't reach the steady state and the trim condition.
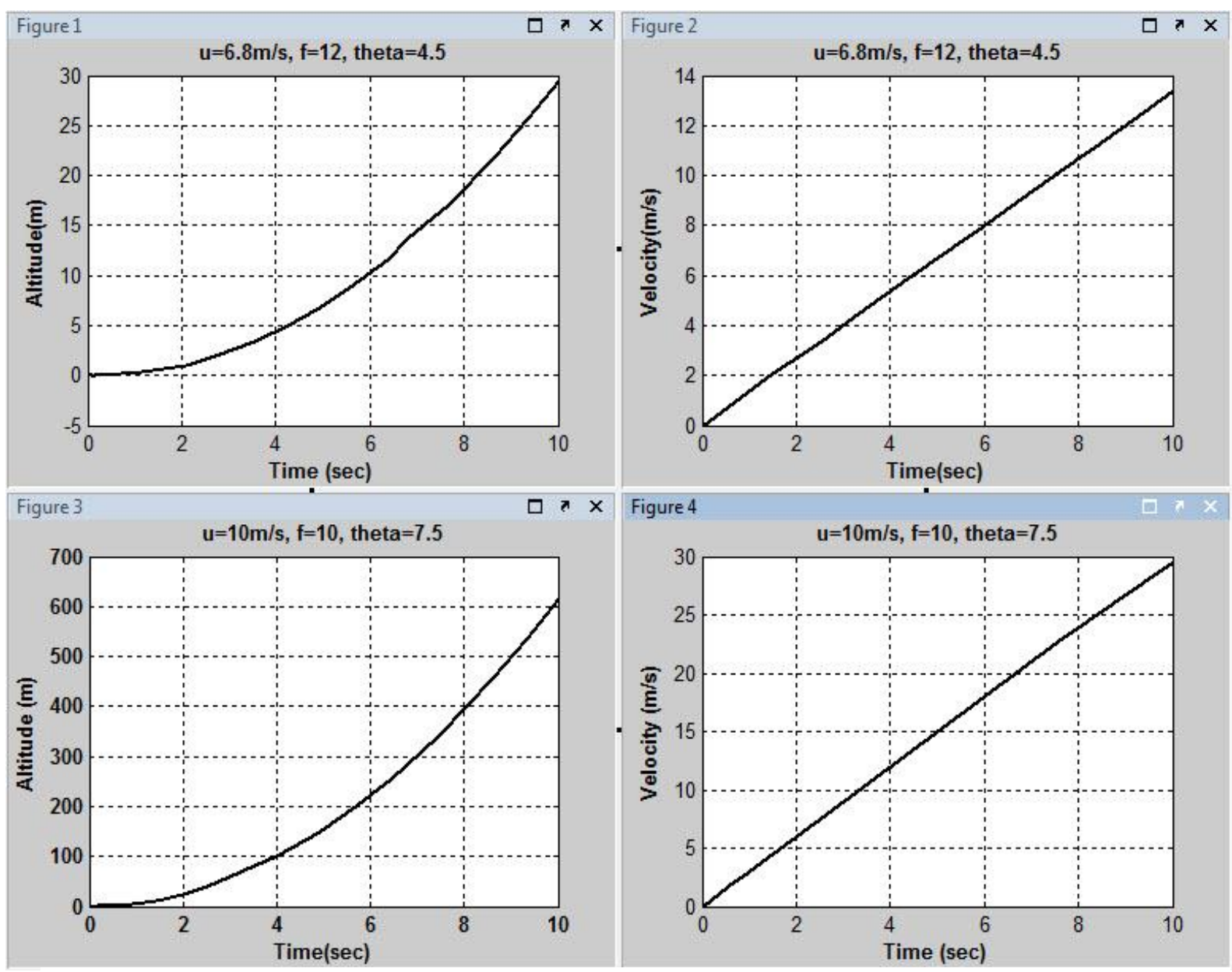

Fig.11. Unsuccessful tries to obtain trim condition without impulsive force for different values of forward velocity, frequency and pitch angles. 


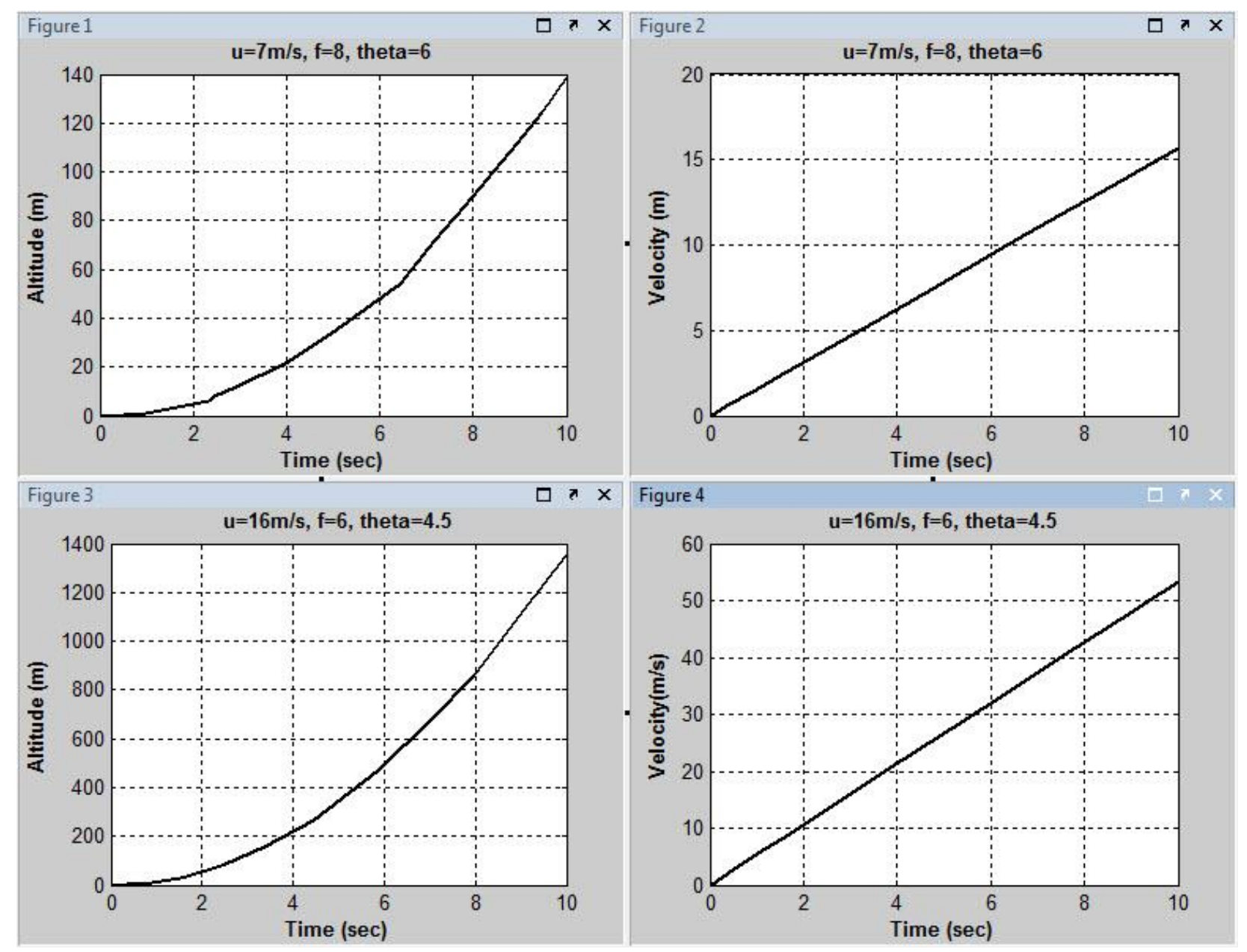

Fig.12. Unsuccessful tries to obtain trim condition without impulsive force for different values of forward velocity, frequency and pitch angles.

\section{Conclusion}

By using efficient multi-body dynamics software MSC.ADAMS ${ }^{\circledR}$ and a refined aerodynamics model, a small flapping air vehicle can be simulated accurately showing the flapping flight performance. Iterations of flight parameters values are made to obtain the trim condition to have a stable flight performance with constant velocity and small fluctuations of pitch angle and altitude. The trim condition is obtained successfully about five times by using an impulse force with different values $100,10000,20000,50000$ and 60000 $\mathrm{N}$. Achieving the trim condition enables the designer to examine the design parameters of the vehicle during realistic flight performance as the aerodynamic model is useful only if the flight speed is constant or near constant.

\section{References}

[1]. Shyy, W., Lian, Y., Tang, J., Viieru, D., Liu, H., "Aerodynamics of Low Reynolds Number Flyers", Cambridge University Press, NY, 2008.

[2]. Mueller, T.J., "Fixed and Flapping Wing Aerodynamics for Micro Air Vehicle Applications", Reston, 2001.

[3]. DeLaurier, J.D., "An Aerodynamic Model for Flapping-Wing Flight", Aeronautical Journal, Vol. 97, pp 125-130, April 1993.

[4]. Kim D K, Lee J S, Lee J Y, Han J H., "An aeroelastic analysis of a flexible flapping wing using modified strip theory", Proceedings of SPIE, San Diego, USA, pp 1-10, 2009. 
[5]. Jones, R.T., "The unsteady lift of a wing of finite aspect ratio", NACA Report No. 681, pp31-38, 1940.

[6]. Kuethe, A.M, Chow C.Y., "The finite wing, foundations of aerodynamics", 4thEd, John Wiley, NY, pp145-164, 1986.

[7]. Scherer, J.O., "Experimental and theoretical investigation of large amplitude oscillating foil propulsion systems", Hydronautics. Laurel, Md. 1968.
[8]. GARRICK, I.E., "propulsion of a flapping and oscillating aerofoil", NACA Report 567, 1936.

[9]. Hoerner, S.F., "Skin-friction drag, fluid dynamics drag", Brick Town Journal, pp2-16. 1965.

[10]. Prouty, R.W. "Airfoils for rotor blades, Helicopter performance, stability and control", Pws Engineering, Boston, 1986. 